\title{
Efficacite Du Baume De Cajou Contre Les Chenilles Carpophages Du Cotonnier Au Nord Du Benin
}

\section{Saturnin Azonkpin}

Centre de Recherches Agricoles - Coton et Fibres (CRA-CF), Institut National des Recherches Agricoles du Bénin (INRAB), Cotonou, Bénin.

\section{Daniel Chèpo Chougourou}

Laboratoire de Recherche en Biologie Appliquée (LARBA), Département de Génie de l'Environnement, Ecole Polytechnique d'Abomey-Calavi (EPAC), Université d'Abomey-Calavi (UAC), Bénin.

\section{Aimé H. Bokonon-Ganta}

Laboratoire d'Entomologie Agricole (LEAg), Faculté des Sciences Agronomiques (FSA), Université d'Abomey-Calavi (UAC), Bénin.

\section{Joseph Dossou}

Laboratoire de Bioingénierie des Procédés Alimentaires

Faculté des Sciences Agronomiques (FSA),

Université d'Abomey-Calavi (UAC)

\section{Léonard E. Ahoton}

Laboratoire de Biologie Végétale, Faculté des Sciences Agronomiques (FSA), Université d'Abomey-Calavi (UAC), Bénin.

\section{Mohamed M. Soumanou}

Laboratoire d’Etude et de Recherche en Chimie Appliquée (LERCA),

Département de Génie de Technologie Alimentaire (DGTA),

Ecole Polytechnique d'Abomey-Calavi (EPAC), Université d'Abomey-Calavi (UAC), Bénin.

\section{Simplice D. Vodouhe}

Laboratoire de Sociologie et de Vulgarisation Agricoles (LSVR), Faculté des Sciences Agronomiques (FSA), Université d'Abomey-Calavi (UAC), Bénin.

Doi: 10.19044/esj.2018.v14n24p464 URL:http://dx.doi.org/10.19044/esj.2018.v14n24p464

\begin{abstract}
Cotton remains the main source of foreign exchange for Benin. This study aims to contribute to increased yield of organic cotton through the evaluation of the effectiveness of cashew balm as an alternative method of management of carpophagous pests of cotton. The experimental setup consists of Fisher Blocks with 6 objects in 4 repetitions. The objects compared are " Unprocessed ", the Agri-bio-pesticide dosed at $11 /$ ha, two concentrations (1\%
\end{abstract}


and $2 \%$ ) of the hot and cold-extracted cashew balm. Linear mixed or fixed effects models and generalized linear models with mixed or fixed effects were used to determine the influence of the objects on the carpophagous larva and their damage in 2015 and 2016 in Thya to the north of Benin. The number of Helicoverpa armigera larva found in 30 plants ranged from 1.2 (Agri-biopesticide) to 2.3 (Untreated) in 2015. The percentages of green capsules with holes varied by $36.08 \%$ (Agri-bio-pesticide) at $53.68 \%$ (1\% cold-extracted balm) in 2015. The percentages of ripe capsules with holes varied from $32.05 \%$ (hot-pressed balm at $2 \%$ ) to $45.00 \%$ (Untreated) in 2016 . Seed cotton yield ranged from $229.91 \mathrm{~kg} / \mathrm{ha}$ (Untreated) to $500 \mathrm{~kg} / \mathrm{ha}$ (Agri-biopesticide) in 2016. It is noted that Agri-bio-pesticide and the $2 \%$ dose of cashew balm, were more effective than that of $1 \%$. The cashew balm is positioned as an alternative to biopesticides used in organic cotton farming.

Keywords: Carpophagous worms, damage, Cashew Nut Shell Liquid, biopesticide, organic cotton, Benin

\section{Resume}

Le coton demeure la principale source d'entrée de devises pour le Bénin. Cette étude vise à contribuer à l'augmentation du rendement en culture de coton biologique, à travers l'évaluation de l'efficacité du baume de cajou contre les ravageurs carpophages du cotonnier. Le dispositif expérimental est constitué des Blocs de Fisher avec 6 objets en 4 répétitions. Les objets comparés sont 'Non Traité', l'Agri-bio-pesticide dosé à 1 1/ha, deux concentrations $(1 \%$ et $2 \%$ ) du baume de cajou extrait à chaud et à froid. Les modèles linéaires à effets mixtes ou fixes et les modèles linéaires généralisés à effets mixtes ou fixes ont été utilisés pour déterminer l'influence des objets sur les chenilles carpophages et leurs dégâts en 2015 et 2016 à Thya au Nord du Bénin. Le nombre de chenilles de Helicoverpa armigera recensées sur 30 plants a varié de 1,2 (Agri-bio-pesticide) à 2,3 (Non Traité) en 2015. Les pourcentages de capsules vertes trouées ont varié de 36,08\% (Agri-biopesticide) à 53,68\% (Baume extrait à froid dosé à 1\%) en 2015. Les pourcentages de capsules mûres trouées ont varié de 32,05\% (Baume extrait à chaud dosé à 2\%) à 45,00\% (Non Traité) en 2016. Le rendement de coton graine a varié de 229,91 kg/ha (Non Traité) à 500 kg/ha (Agri-bio-pesticide) en 2016. On note que Agri-bio-pesticide et la dose de $2 \%$ du Baume de cajou, ont été plus efficaces que celle de $1 \%$. Le baume de cajou se positionne comme une alternative aux biopesticides pouvant être utilisé en culture cotonnière biologique.

Mots clés : Chenilles carpophages, dégâts, baume de cajou, biopesticide, coton biologique, Bénin 


\section{Introduction}

Le coton constitue la principale source de revenu pour plus de deux millions de producteurs et contribue pour environ 25 à $45 \%$ des revenus d'exportation des pays producteurs (Baffes, 2007 ; Moseley et Gray, 2008). Le coton organique est actuellement produit dans 22 pays dans le monde, couvrant les mêmes régions que celles du coton conventionnel (Ferrigno et Lizarraga, 2009). En Afrique, l'agriculture biologique certifiée est un phénomène encore récent. Les produits biologiques africains qui sont exportés sont les fruits, les légumes, le café, le cacao et le coton (Diakite et Djouara, 2003). Huit pays (Bénin, Burkina Faso, Mali, Sénégal, Afrique du Sud, Tanzanie, Ouganda et Zambie) continuent d'être les chefs de file de la production du coton organique sur le continent. La Tanzanie et l'Ouganda sont les plus grands producteurs africains de coton organique, représentant $83 \%$ de la production totale de fibres en Afrique (Ferrigno et Lizarraga, 2009).

A l'instar de la plupart des pays africains, le Bénin a une économie basée sur l'agriculture. Parmi les spéculations, le coton demeure la principale source d'entrée de devises pour le pays. La promotion de la production et la commercialisation du coton biologique certifié au Bénin remonte à 1996 avec l'Organisation Béninoise pour la Promotion de l'Agriculture Biologique (OBEPAB). Le Projet d'Appui au Développement de la Commune de Kandi (PADEC) intervenait aussi pour la même cause dans cette Commune située au Nord du Bénin. Mais, ces dernières années, on assiste dans la sous-région à une stagnation des rendements, voire même une régression qui pourrait s'expliquer par l'épuisement des sols, une recrudescence du parasitisme, une mauvaise application des itinéraires techniques de production et la cherté des intrants chimiques (FAO, 2014). Il en est de même au niveau des rendements de la production du coton-graine biologique qui demeurent faibles. Tovignan (2012) a rapporté qu'au cours de la campagne agricole 2010-2011, le rendement du coton-graine biologique a varié de $360 \mathrm{~kg} / \mathrm{ha}$ (dans la zone Alafia au Nord-ouest du Bénin) à $540 \mathrm{~kg} / \mathrm{ha}$ (dans la zone encadrée par l'Organisation Béninoise pour la Promotion de l'Agriculture Biologique $(\mathrm{OBEPAB}))$. La production du coton-graine biologique est aussi confrontée à quelques contraintes qui limitent son évolution. Parmi ces contraintes figurent la faible disponibilité des intrants agricoles, la mauvaise qualité et la faible efficacité des intrants utilisés sur le coton biologique qui contribuent énormément au faible niveau des rendements (OBEPAB, 2002). Au nombre de ces contraintes, celles relatives à la protection phytosanitaire des cotonniers occupent une place prépondérante. En effet, la culture cotonnière subit d'importants dégâts provoqués par un large complexe de ravageurs comprenant une centaine d'arthropodes (Kranthi et al., 2001). Parmi les ravageurs du cotonnier qui causent plus de dégâts au Bénin figurent les ravageurs carpophages tels que Helicoverpa armigera, Earias spp., 
Diparopsis watersi, Spodoptera littoralis, Pectinophora gossypiella, Thaumatotibia leucotreta. Selon Ferron et al. (2006), la pression parasitaire induit une perte de récolte de l'ordre de $20 \%$. La faune entomologique constitue donc l'un des facteurs les plus importants dans les baisses de productivité et des rendements. La maîtrise des arthropodes ravageurs représente donc une opération importante dans la production cotonnière (Matthews, 1989).

Ainsi, des recherches méritent d'être approfondies pour améliorer les technologies développées en matière de protection phytosanitaire en culture du coton biologique afin d'améliorer le rendement. Il est donc nécessaire de favoriser le développement des insecticides naturels à partir des plantes, tel que le baume de cajou encore appelé Cashew Nut Shell Liquid (CNSL). Ce produit issu de la coque de la noix de cajou, possède des propriétés biocides intéressantes sur certains nuisibles (Cavalcante et al., 2003; Chabi et al., 2013; Chabi et al., 2014) ) et en particulier sur les ravageurs de niébé au champ et en stock (Kpoviessi et al 2017). L'usage des plantes pesticides se révèle être une pratique ancestrale en Afrique (Yarou et al, 2017). De nombreuses plantes sont connues et utilisées pour leurs activités biocides (toxique, répulsive, antiappétante) vis-à-vis d'une large gamme de bioagresseurs (Yarou et al, 2017). Malgré ces propriétés connues des organes de la plante, aucune étude n'a encore exploré les effets biocides du baume de cajou sur les ravageurs carpophages du cotonnier dans les parcelles de culture. La présente étude vise à évaluer l'effet insecticide du baume de cajou contre les ravageurs carpophages du cotonnier au champ. Spécifiquement, il s'agit de déterminer l'influence de deux modes d'extraction et de deux doses de baume de cajou sur la densité des chenilles carpophages et leurs dégâts sur le cotonnier au champ.

\section{Materiel et Methodes \\ Zone d'étude}

La présente étude a été menée en 2015 et 2016 au niveau du site de Thya situé dans l'arrondissement d'Angaradébou à Kandi (Figure 1). Le choix de ce site situé au Nord du Bénin est guidé par les critères de l'importance du volume produit et l'ancienneté dans la production du coton biologique. Cette Commune fait partie des grandes zones de production du coton biologique au Bénin (Hougni et al., 2012). 


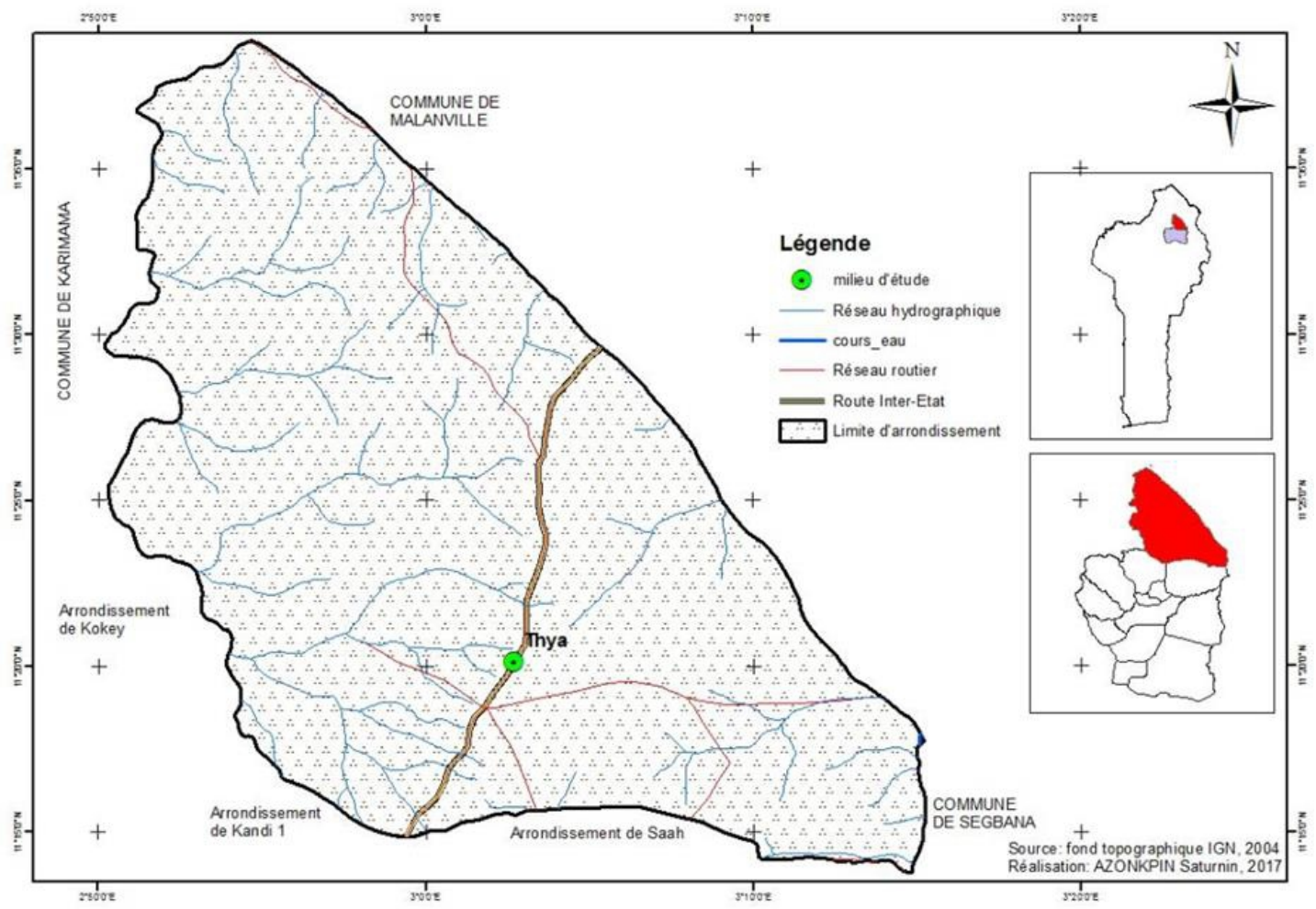

Figure 1 : Situation et présentation de la zone d'étude

\section{Matériel}

Le matériel végétal utilisé est la variété de cotonnier ANG 956 créée par le Centre de Recherches Agricoles - Coton et Fibres (CRA-CF). Cette variété a succédé à la variété $\mathrm{H} 279-1$ qui était cultivée dans la zone depuis plusieurs années. Deux types de biopesticides ont été utilisés. Il s'agit du témoin de référence, Agri-Bio-Pesticide qui est actuellement utilisé par la plupart des producteurs de coton biologique au Bénin et du Baume de cajou extrait à chaud ou à froid. L'Agri-Bio-Pesticide est un pesticide biologique à base de graines de neem, du savon indigène "koto" et du piment pili-pili. Selon Deravel et al. (2013), l'huile extraite des graines de neem contient plusieurs molécules biologiquement actives dont l'azadirachtine, la nimbidine, la nimbidinine, la solanine, le déacétylazadirchtinol et le méliantriol. De plus, l'activité biocide des alcaloïdes, des saponines et des flavonoïdes extraits des fruits de Capsicum frutescens $\mathrm{L}$. a été confirmée par Bouchelta et al. (2005). Quant au baume de cajou, sa composition chimique varie en fonction des méthodes d'extraction qui peuvent être à froid ou à chaud (Araùjo et Xavier, 2009). Selon ces auteurs, tous les deux types de baume comportent principalement de l'acide anacardique, du cardanol, du cardol, du 2-méthylcardol à des proportions différentes. 


\section{Méthodes}

Cette expérimentation a été conduite pour tester l'effet de deux doses de baume de cajou extrait à chaud et à froid sur les principaux insectes ravageurs carpophages du cotonnier au Nord du Bénin.

\section{Dispositif expérimental}

Le dispositif expérimental est constitué des Blocs de Fisher avec 6 objets en 4 répétitions et des parcelles élémentaires de 8 lignes de $9 \mathrm{~m}$ de longueur dont 6 traitées.

Les objets comparés dans cet essai et leurs caractéristiques sont décrits dans le tableau 1.

Tableau 1 : Objets comparés et leurs doses

\begin{tabular}{|l|c|}
\hline Objets / bio-insecticides et concentrations & Dose (L/ha) \\
\hline A- Non Traité & - \\
B- Agri-bio-pesticide & 1 \\
C- Baume de cajou extrait à chaud 2\% & 0,2 \\
D- Baume de cajou extrait à chaud 1\% & 0,1 \\
E- Baume de cajou extrait à froid 2\% & 0,2 \\
F- Baume de cajou extrait à froid 1\% & 0,1 \\
\hline
\end{tabular}

Quatorze (14) applications de chaque objet ont été réalisées entre les $31^{\text {ème }}$ et $122^{\text {ème }}$ jours après la levée (j.a.l) de la culture avec une périodicité de 7 jours à l'aide d'un appareil à dos de type Solo 425 qui est un pulvérisateur manuel à dos à pression entretenue contenant 1,7 litres d'eau plus la quantité de produit pour traiter chaque objet aux $31,38,45,52,59,66,73,80,87,94$, 101, 108, 115, 122 j.a.l. L'Agri-bio-pesticide a été obtenu auprès d'une structure de production sise à Aglomè dans la Commune de Djidja. Le Baume de cajou a été extrait à chaud et à froid au Laboratoire de Bio ingénierie et de Production Alimentaire de la Faculté des Sciences Agronomiques à l'Université d'Abomey-Calavi. Lors de la préparation de la bouillie de Baume de cajou, on a ajouté au contenu du pulvérisateur, du savon liquide Mir multiusages utilisé comme un émulsifiant (adjuvant) à la même dose que le produit.

\section{Données collectées}

Dans les conditions d'expérimentation en champ, l'efficacité des extraits de plantes est généralement mesurée à travers l'abondance des populations des ravageurs ou la sévérité des dégâts (Yarou et al., 2017). Ainsi, au niveau de l'évaluation de la densité des ravageurs, nous avons collecté les données suivantes :

- les chenilles carpophages (Helicoverpa armigera, Earias spp., Diparopsis watersi, Pectinophora gossypiella, Thaumatotibia leucotreta) ont été dénombrées à la veille de chaque traitement, soit au 30, 37, 44, 51, 58, 65, 72, 79, 86, 93, 100, 107, 114 et 121 j.a.l, sur 30 plants par parcelle élémentaire 
pris par groupe de 5 plants de façon consécutive sur les lignes centrales, selon la méthode de la diagonale du $30^{\text {ème }}$ au $121^{\text {ème }}$ j.a.l des cotonniers ;

- les chenilles carpophages (Helicoverpa armigera, Earias spp., Diparopsis watersi, Pectinophora gossypiella, Thaumatotibia leucotreta) ont été dénombrées de façon hebdomadaire sur 50 capsules vertes de même âge (diamètre supérieur à $2 \mathrm{~cm}$ ) par parcelle élémentaire sur les lignes 2 et 7 à partir du $80^{\text {ème }}$ jusqu'au $115^{\text {ème }}$ j.a.l.

Au niveau de l'évaluation des dégâts des ravageurs, nous avons collecté les données suivantes :

- les capsules vertes ont été dépouillées afin de dénombrer les capsules saines ou attaquées (trouées, parasitées et piquées). Cette analyse sanitaire des capsules vertes a été réalisée de façon hebdomadaire sur 50 capsules vertes de même âge (diamètre supérieur à $2 \mathrm{~cm}$ ) par parcelle élémentaire sur les lignes 2 et 7 à partir du $80^{\text {ème }}$ jusqu'au $115^{\text {ème }}$ j.a.l.

- les capsules mûres ont été dépouillées afin de dénombrer les capsules saines et attaquées (trouées, piquées, pourries et momifiées). Cette analyse sanitaire des capsules mûres a été réalisée à la récolte sur la ligne 3 sur une séquence de 7 m délimitée au centre de la ligne ;

- le rendement du coton graine a été évalué après la récolte en 2 passages sur les lignes 4 et 5 ;

$$
\text { Rendement }=\frac{\text { Poids cumulé } d u \text { coton des } 2 \text { lignes }(\mathrm{kg}) \times 10000 \mathrm{~m} 2}{2 \text { lignes } \times 9 \mathrm{~m} \times 0,80 \mathrm{~m}}
$$

- la densité des plants a été évaluée en comptant les plants présents à la récolte sur les lignes 4 et 5 .

\section{Méthode d'analyse des données}

Afin de tester l'effet «traitement» (facteur fixe) et celui du bloc (facteur aléatoire) sur les nombres de chenilles carpophages retrouvés sur les cotonniers; les pourcentages de capsules vertes saines et attaquées; les pourcentages de capsules mûres saines et attaquées; la densité des plants et enfin le rendement, différents modèles ont été utilisés. Il s'agit des modèles linéaires généralisés à effets mixtes, les modèles linéaires généralisés à effets fixes, les modèles linéaires à effets mixtes et des modèles linéaires à effets fixes. Pour chaque catégorie de variable réponse, différents modèles à effets mixtes sont établis et testés (modèle complet, modèle à intercept aléatoire, modèle à pente aléatoire puis modèle à pente aléatoire et intercept aléatoire). Le meilleur est retenu sur la base de l'AICc le plus faible (Burnham et Anderson, 2002). Au cas, où il est observé la non significativité de l'effet bloc (Prob. $>0,05$ ou ICC $<50 \%$ ), le nouveau modèle établit est un modèle croisé fixe. Quand les effets individuels sont significatifs, la structuration des moyennes est faite afin d'identifier les meilleurs groupes de traitement qui ont 
des effets significatifs sur les ravageurs ou leur dégâts en culture cotonnière biologique au Benin.

Le logiciel R Studio 3.3.3 (R Development Core Team, 2017) a été utilisé pour le traitement des données collectées. En effet, les fonctions ' 'glmmadmb' et ''glmmPQL', du package ''glmmADMB', (Bolker et al, 2012) dans le cadre des modèles linéaires généralisés à effets mixtes ont été utilisées pour voir l'existence d'une différence significative des facteurs traitement et bloc suivant le nombre de chenilles (distribution de poisson et ses extensions ont été testées) ; le pourcentage de capsules vertes et mûres, saines ou attaquées (distribution bêta) dans le temps. Puis la fonction 'Ime', du package " nlme'" (Pinheiro et al., 2017) dans le cadre des modèles linéaires à effets mixtes est utilisée pour ce qui concerne le rendement (distribution normale). La significativité des facteurs fixes a été évaluée avec la fonction 'Anova', du package ' car', (Fox and Weisberg, 2011) et celle du facteur aléatoire sur la base du calcul des Corrélations InterClasse (ICC). Préalablement, le choix du meilleur modèle a été fait sur la base des résultats produits avec la fonction "AICctab" du package "'bbmle' (Burnham et Anderson, 2002). La réalisation des modèles linéaires à effets fixes a impliqué deux cas : (i) cas des modèles linéaires généralisés à effets fixes où la fonction " glm' du package "'MASS' (Venables et Ripley, 2002) a été utilisé et (ii) le cas des modèles linéaires à effets fixes avec l'usage la fonction ' $1 \mathrm{~m}$ ' du packages "stats" par défaut. En cas de différence significative observée au seuil de $5 \%$, un post-hoc test relatif à la méthode de Tukey a été fait pour comparaison multiple de moyennes ajustées du facteur traitement, avec le package 'lsmeans' (Russell, 2016), puis représenté sous forme graphique. Les barres affectées d'une lettre identique ne sont pas statistiquement différents pour tous les traitements. De même, les valeurs possédant la même lettre ne sont pas significativement différentes.

\section{Resultats}

\section{Evaluation de la densité des ravageurs}

\section{Dénombrement des chenilles de Helicoverpa armigera sur les plants}

La figure 2 présente les résultats de dénombrement des chenilles de $H$. armigera sur les cotonniers à Thya. Ces résultats ont été très hautement significatifs à $0,1 \%$ au niveau du nombre moyen de $H$. armigera $(\mathrm{P}=4,522 \mathrm{e}-$ 12). Le nombre de chenilles de $H$. armigera dénombré en 2015 a été plus élevé que celui de 2016. Le nombre de chenilles de $H$. armigera a varié respectivement en 2015 et 2016 de 1,2 chenille (Agri-bio-pesticide) à 2,3 chenilles (Non Traité) et de 0,77 chenille (Agri-bio-pesticide) à 1,8 chenille (Non Traité). Tous les biopesticides ont mieux réduit le nombre de chenilles de $H$. armigera par rapport au témoin absolu 'Non Traité', durant les deux années d'expérimentation. Si en 2015 il n'existe pas de différence significative 
entre les biopesticides comparés, en 2016 Agri-bio-pesticide a mieux réduit le nombre de chenilles de $H$. armigera par rapport au Baume de cajou extrait à chaud dosé à $1 \%$ et au Baume de cajou extrait à froid dosé à $1 \%$. Le Baume de cajou extrait à froid comme à chaud dosé à $2 \%$ a eu la même performance sur les chenilles de $H$. armigera que le témoin de référence Agri-bio-pesticide.

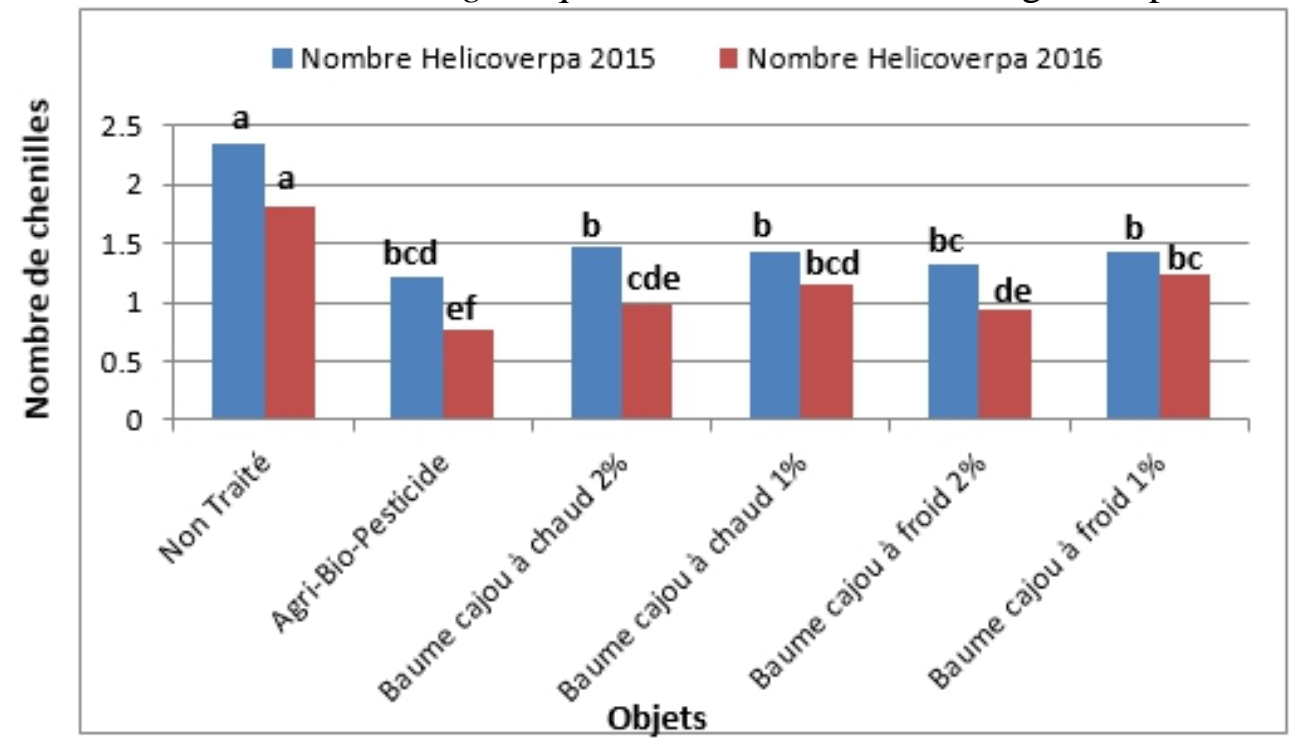

Figure 2 : Nombre de chenilles de Helicoverpa sur 30 plants à Thya

\section{Dénombrement de chenilles de Helicoverpa armigera dans les capsules vertes}

La figure 3 présente les résultats de dénombrement des chenilles de Helicoverpa armigera dans cent (100) capsules vertes. Ces résultats ont été discriminants au seuil de $0,1 \%(\mathrm{P}=2,2 \mathrm{e}-16)$. Le nombre de chenilles de $H$. armigera présentes dans les capsules vertes a varié de 0,04 (Baume de cajou extrait à froid dosé à $2 \%$ ) à 0,15 (Non Traitées) en 2015 . Ce nombre a varié de 0,02 (Baume de cajou extrait à chaud dosé à $2 \%$ ) à 0,1 (Non Traités) en 2016. Les différentes concentrations de Baume de cajou extrait à froid comme à chaud ont mieux réduit le nombre de chenilles de $H$. armigera par rapport au 'Non Traité' et Agri-bio-pesticide en 2015. Mais en 2016, ces concentrations de Baume de cajou ont présenté les mêmes performances que le témoin de référence Agri-bio-pesticide. Le mode d'extraction du Baume de cajou n'a pas eu d'influence sur le nombre de chenilles de $H$. armigera. 


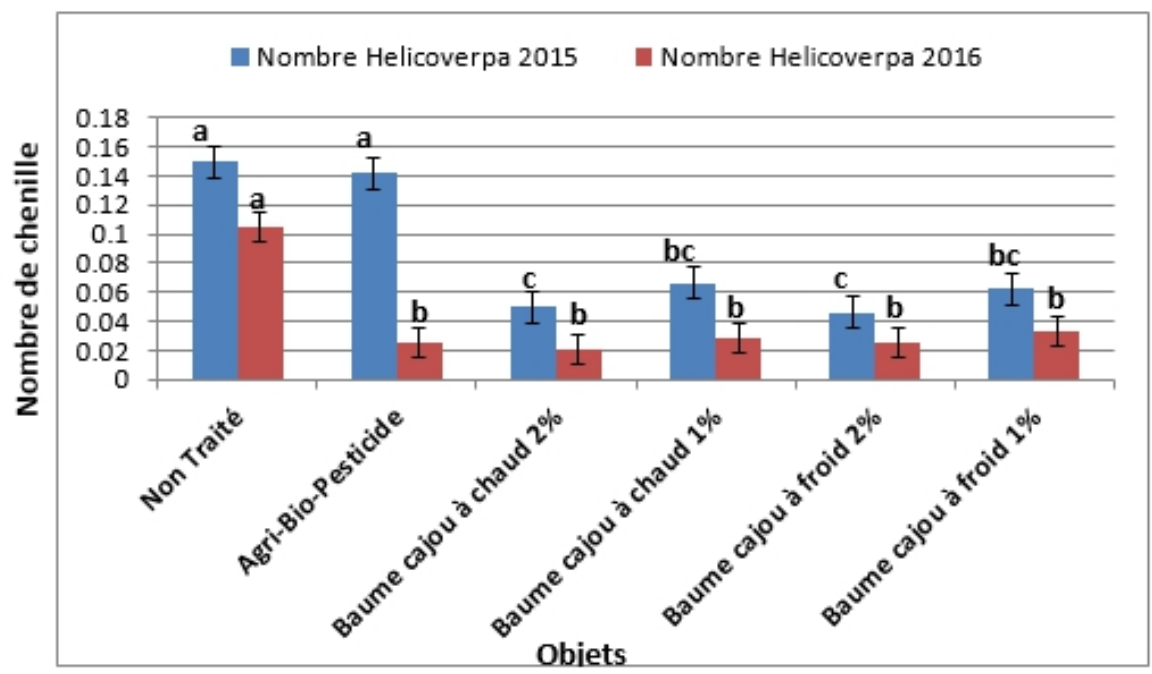

Figure 3 : Nombre de chenilles de Helicoverpa armigera dans les capsules vertes

\section{Dénombrement de chenilles de Diparopsis watersi sur les plants}

La figure 4 montre les résultats de dénombrement des chenilles de $D$. watersi sur les cotonniers à Thya. Ces résultats ont été très hautement significatifs à $0,1 \%$ au niveau du nombre moyen de D. watersi $(\mathrm{P}=5.467 \mathrm{e}$ 06). Le nombre de chenilles de D. watersi varie de 0,4 chenille (Agri-biopesticide et Baume de cajou à froid dosé à $1 \%$ ) à 1,16 chenille (Non Traité) en 2015, puis de 0,98 chenille (Agri-bio-pesticide) à 1,6 chenille (Non Traité) en 2016. Les biopesticides ont réduit significativement le nombre de chenilles de D. watersi par rapport au témoin 'Non Traité' en 2015. Mais comparés au témoin de référence Agri-bio-pesticide, les deux concentrations de baume de cajou extrait à chaud comme à froid ont eu une efficacité similaire au témoin de référence durant les deux années d'expérimentation.

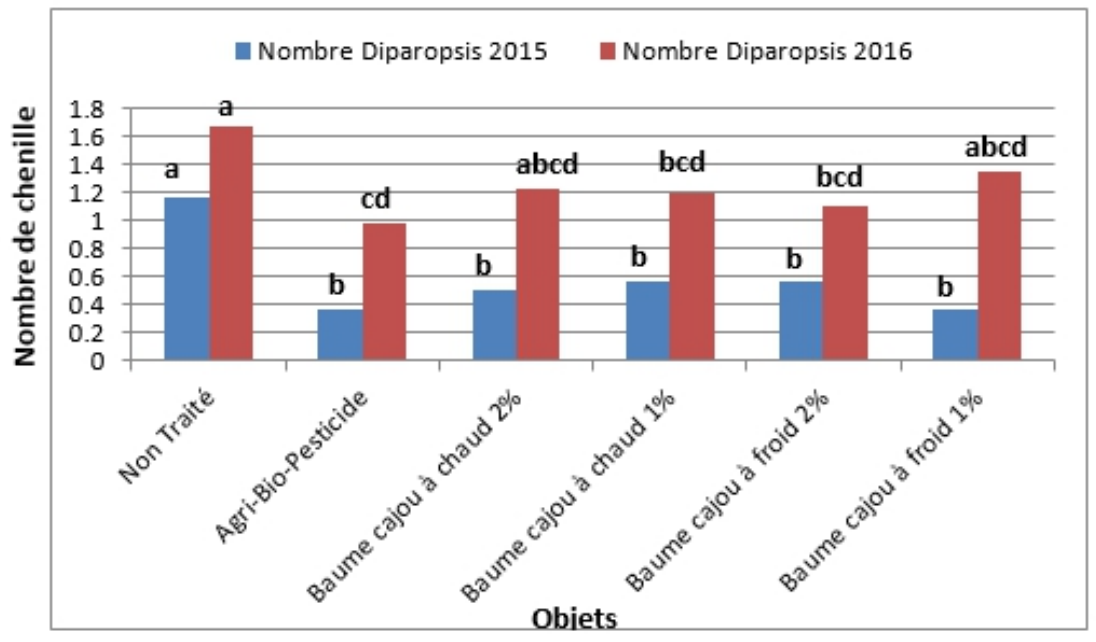

Figure 4 : Nombre de chenilles de Diparopsis watersi sur 30 plants à Thya 
Les résultats du dénombrement des chenilles d'Earias spp. et de Spodoptera littoralis présentes sur 30 plants, n'ont pas été discriminants. Les chenilles de Pectinophora gossypiella et de Thaumatotibia leucotreta ont été absentes dans cet essai. Ils n'ont pas été rencontrés au cours des observations de dénombrement de chenilles sur plants à Thya.

\section{Dénombrement de chenilles de Diparopsis watersi dans les capsules vertes}

La figure 5 présente les résultats de dénombrement des chenilles de $D$. watersi dans cent (100) capsules vertes en 2016. Ces résultats ont été très hautement significatifs à $0,1 \%$ au niveau du nombre moyen de chenilles. Le nombre de chenilles de $D$. watersi présentes dans les capsules vertes varie de 0,04 chenille (Agri-bio-pesticide) à 0,10 chenille (Non Traités). En dehors du Baume de cajou extrait à froid dosé à $1 \%$, tous les autres biopesticides ont mieux réduit le nombre de chenilles de D. watersi par rapport au 'Non Traité'. Mais il n'existe pas de différence significative entre les différentes concentrations de baume de cajou qui ont présenté une performance similaire au témoin de référence Agri-bio-pesticide sur $D$. watersi. De même, le mode d'extraction du Baume de cajou n'a pas influencé le nombre de chenilles de ce ravageur.

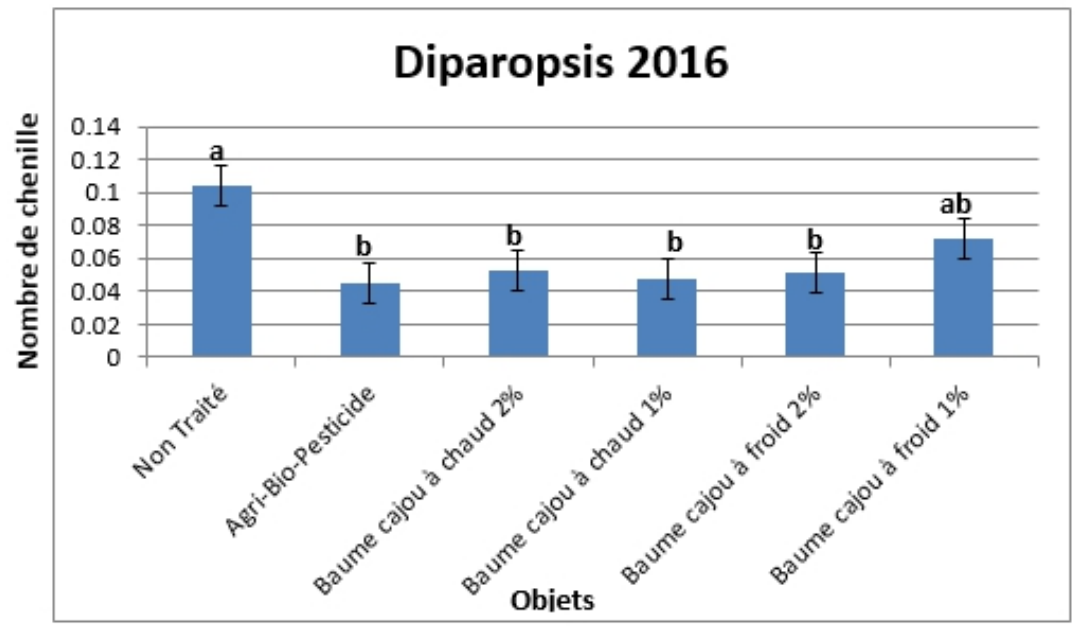

Figure 5: Nombre de chenilles de Diparopsis watersi dans les capsules vertes

\section{Dénombrement de chenilles de Earias spp. dans les capsules vertes}

La figure 6 présente les résultats de dénombrement des chenilles de Earias spp. dans cent (100) capsules vertes en 2016. Ces résultats ont été discriminants au seuil de 0,1\%. Le nombre de chenilles de Earias spp. présentes dans les capsules vertes varie de 0,00 chenille (Baume de cajou extrait à chaud dosé à $1 \%$ ) à 0,09 chenille (Non Traitée). Les différentes concentrations de baume de cajou et Agri-bio-pesticide ont réduit significativement le nombre de chenilles de Earias spp. par rapport au témoin 
'Non Traité". Mais il n'existe pas de différence significative entre les concentrations de baume de cajou et le témoin de référence Agri-bio-pesticide. Le mode d'extraction du Baume de cajou n'a pas eu d'effet sur le nombre de chenilles de Earias spp..

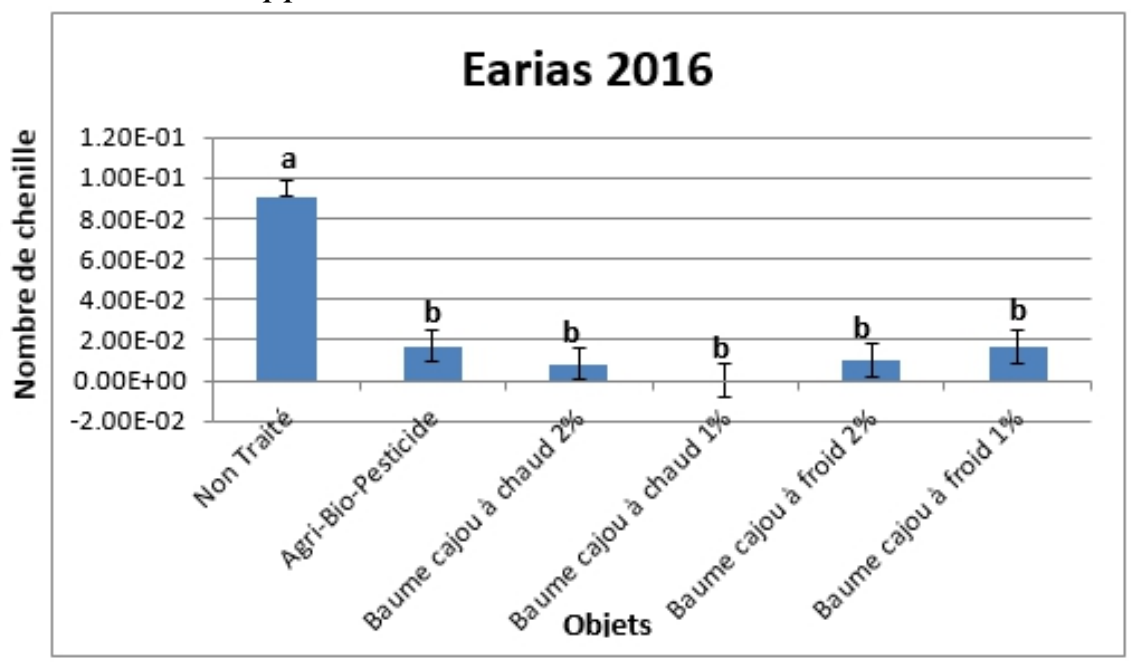

Figure 6 : Nombre de chenilles de Earias spp. dans les capsules vertes

\section{Evaluation des dégâts des ravageurs carpophages Pourcentage de capsules vertes saines}

La figure 7 présente les résultats de dénombrement des capsules vertes saines. Ces résultats ont été discriminants au seuil de $0,1 \%(\mathrm{P}=2,2 \mathrm{e}-16)$. Au moment où $56,19 \%$ de capsules vertes saines ont été obtenues au niveau des parcelles traitées avec le Baume de cajou extrait à chaud dosé à $2 \%$ en 2015, les parcelles 'Non Traitées" n'ont obtenu que 35,24\%. En 2016, les parcelles "Non Traitées"' ont obtenu 26,10\% de capsules vertes saines contre 49,03\% pour les parcelles traitées au Baume de cajou extrait à chaud dosé à $2 \%$. En dehors du Baume de cajou extrait à froid dosé à $1 \%$, tous les autres biopesticides ont amélioré le pourcentage de capsules vertes saines par rapport au témoin " Non Traitées" en 2015. En 2016, tous les biopesticides ont amélioré le pourcentage de capsules vertes saines par rapport au témoin " Non Traitées". Les différentes concentrations de Baume de cajou ont eu la même performance que le témoin de référence, Agri-bio-pesticide durant les deux années d'expérimentation. Mais dosé à $2 \%$, le Baume de cajou extrait à froid a mieux amélioré le pourcentage de capsules vertes saines par rapport à la dose de $1 \%$. La faiblesse de la concentration de $1 \%$ de Baume de cajou extrait à chaud comme à froid a été également observée comme constaté au niveau des chenilles. De même le mode d'extraction n'a pas influencé le pourcentage de capsules vertes saines. 


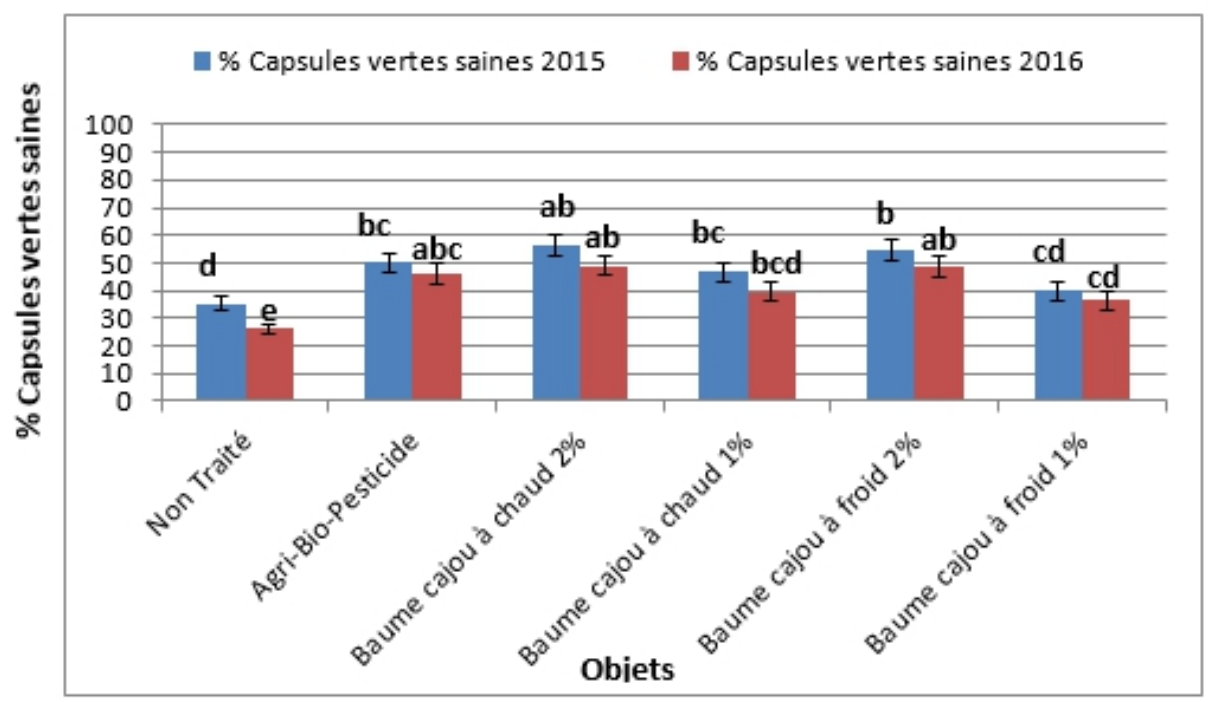

Figure 7 : Pourcentage de capsules vertes saines à Thya

\section{Pourcentage de capsules vertes trouées}

La figure 8 montre les résultats de dénombrement des capsules vertes trouées à Thya. Ces résultats ont été très hautement significatifs à $0,1 \%$ au niveau du pourcentage moyen $(\mathrm{P}=2 \mathrm{e}-16)$. En 2015, le pourcentage des capsules vertes trouées a varié de 36,08\% (Agri-bio-pesticide) à 53,68\% (Baume de cajou extrait à froid dosé à 1\%). En 2016 ces pourcentages ont varié de 32,00\% (Baume de cajou extrait à chaud dosé à $2 \%$ et Baume de cajou extrait à froid dosé à 2\%) à 48,63\% (Non Traité). En 2015, le Baume de cajou extrait à chaud dosé à $2 \%$ et le Baume de cajou extrait à froid dosé à $2 \%$ ont eu la même performance que le témoin de référence Agri-bio-pesticide qui a mieux réduit les capsules vertes trouées par rapport au Baume de cajou extrait à chaud comme à froid et dosé à $1 \%$. Mais en 2016 il n'existe pas de différence significative entre les biopesticides comparés qui ont mieux réduit les capsules vertes trouées par rapport au 'Non traité'. De même, il n'existe pas de différence significative entre les deux modes d'extraction testés. 


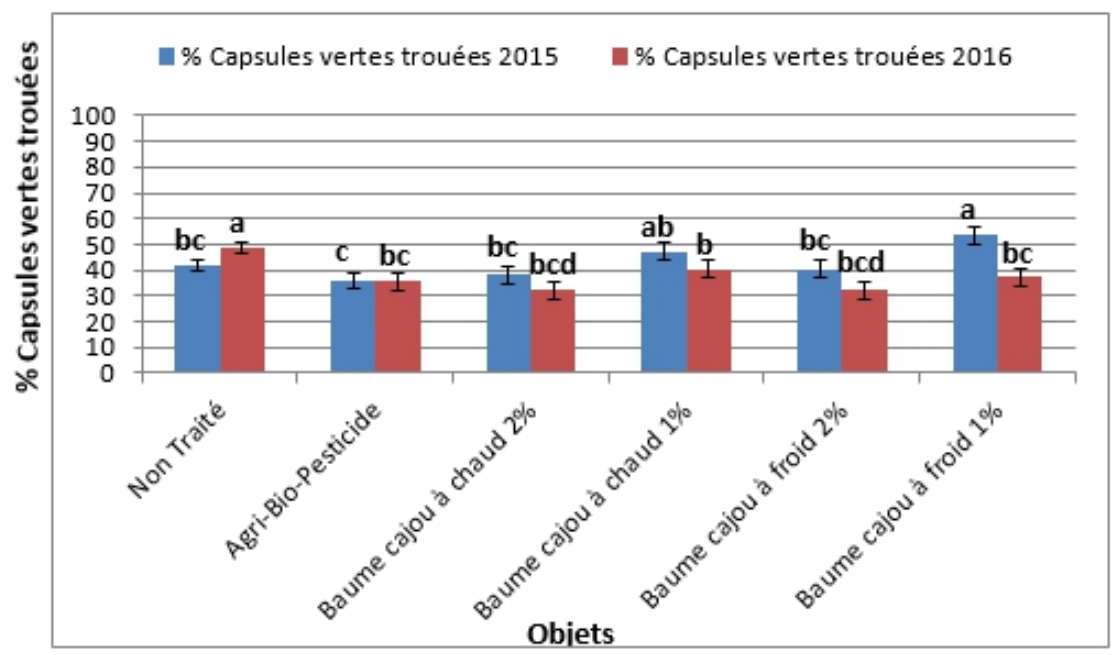

Figure 8: Pourcentage de capsules vertes trouées à Thya

\section{Pourcentage de capsules mûres saines}

La figure 9 présente les résultats de l'analyse sanitaire des capsules mûres saines en 2016. Ces résultats ont été hautement significatifs à $0,1 \%(\mathrm{P}$ $=4,3 \mathrm{e}-10)$. Le Baume de cajou extrait à chaud dosé à $2 \%$ et Agri-bio-pesticide ont permis d'obtenir 40,09\% de capsules mûres saines contre $27,46 \%$ pour le témoin 'Non Traité'. Il n'existe pas de différence significative entre les différentes concentrations de Baume de cajou qui ont présenté une performance similaire au témoin de référence Agri-bio-pesticide. De même, il n'existe pas de différence significative entre les deux modes d'extraction testés.

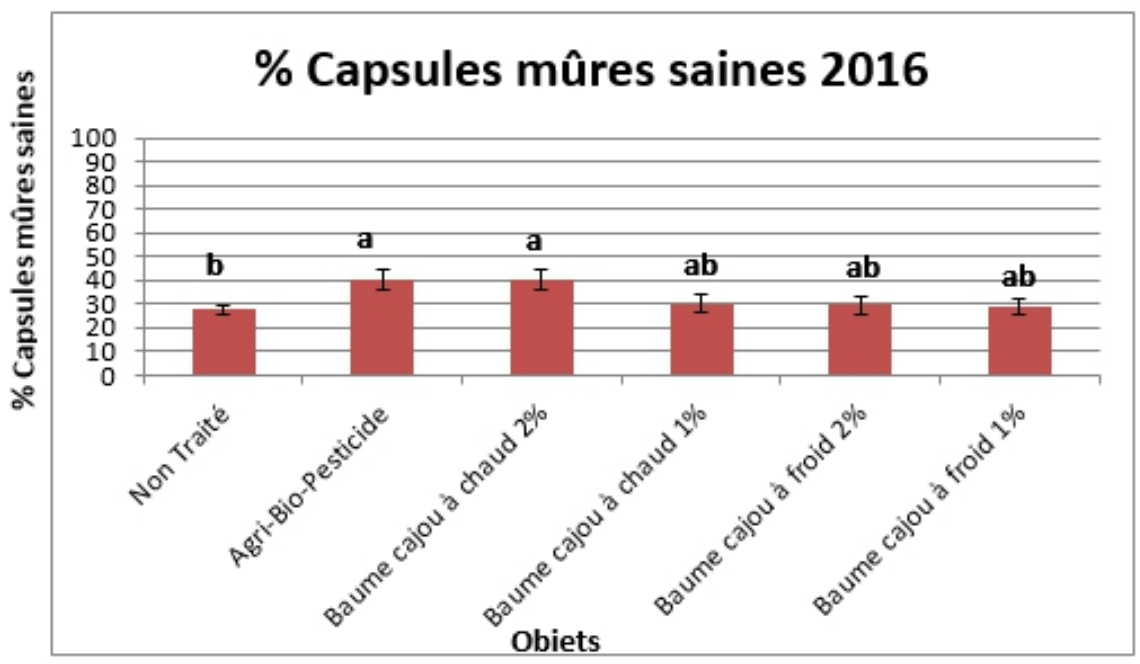

Figure 9 : Pourcentage de capsules mûres saines 


\section{Pourcentage de capsules mûres trouées}

La figure 10 montre les résultats de l'analyse sanitaire des capsules mûres trouées en 2016. Ces résultats ont été hautement significatifs à $0,1 \%$ (P $=2,192 \mathrm{e}-10)$. Au moment où $45,00 \%$ de capsules mûres trouées ont été dénombrées au niveau du témoin 'Non Traité', 32,05\% de ces capsules ont été recensées au niveau du Baume de cajou extrait à chaud dosé à $2 \%$. Il n'existe pas de différence significative entre les différentes concentrations de Baume de cajou qui ont présenté une performance similaire au témoin de référence Agri-bio-pesticide. Le mode d'extraction n'a pas influencé le pourcentage de capsules mûres trouées.

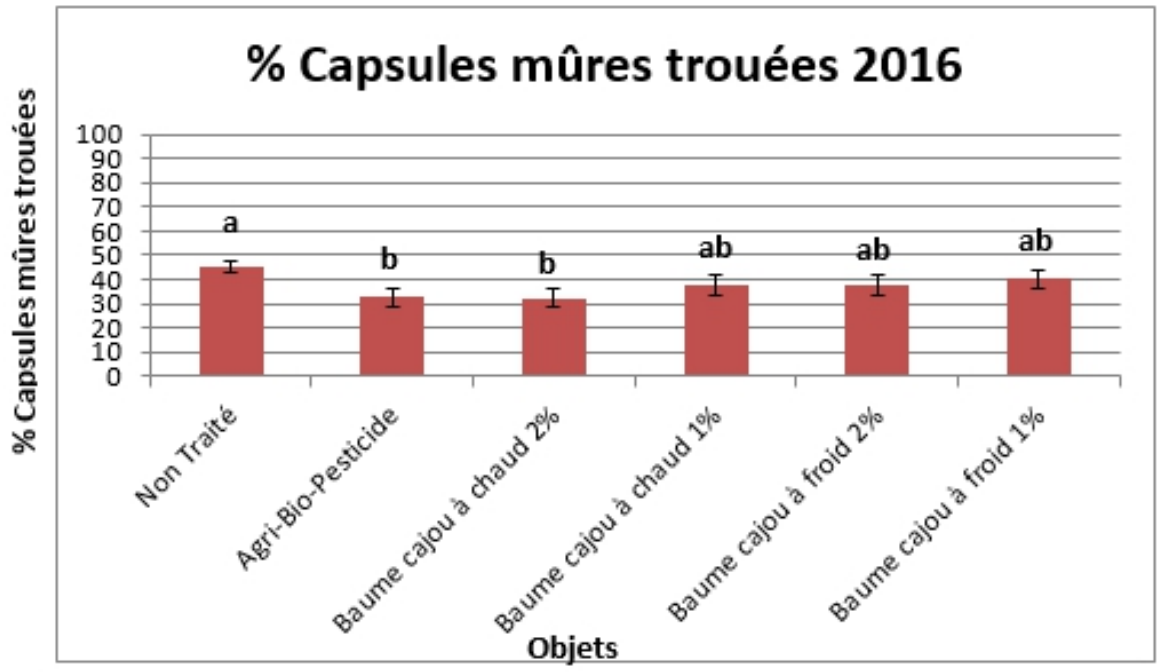

Figure 10: Pourcentage de capsules mûres trouées

\section{Rendement de coton-graine}

La figure 11 présente les résultats de rendement de coton graine obtenu en 2016. Ces résultats ont été très hautement significatifs à $0,1 \%$ au niveau du rendement moyen $(\mathrm{P}=2.2 \mathrm{e}-16)$. Les parcelles traitées avec Agri-bio-pesticide ont permis d'obtenir $500 \mathrm{~kg} /$ ha contre $229,91 \mathrm{~kg} / \mathrm{ha}$ pour les parcelles 'Non Traitées'. En dehors du Baume de cajou extrait à froid dosé à 1\%, toutes les autres concentrations de Baume de cajou et l'Agri-bio-pesticide, ont amélioré le rendement par rapport au témoin 'Non Traité'. L'Agri-bio-pesticide, le Baume de cajou extrait à chaud dosé à $2 \%$ et le Baume de cajou extrait à froid dosé à $2 \%$ ont été les meilleurs biopesticides au niveau du rendement. La concentration de $1 \%$ de Baume de cajou extrait à chaud comme à froid a permis d'obtenir des rendements inférieurs au témoin de référence Agri-biopesticide. La tendance selon laquelle la concentration de $1 \%$ de Baume de cajou extrait à chaud comme à froid serait insuffisante en cas de forte infestation a été confirmé au niveau du rendement. De même, le mode d'extraction n'a pas influencé le rendement. 


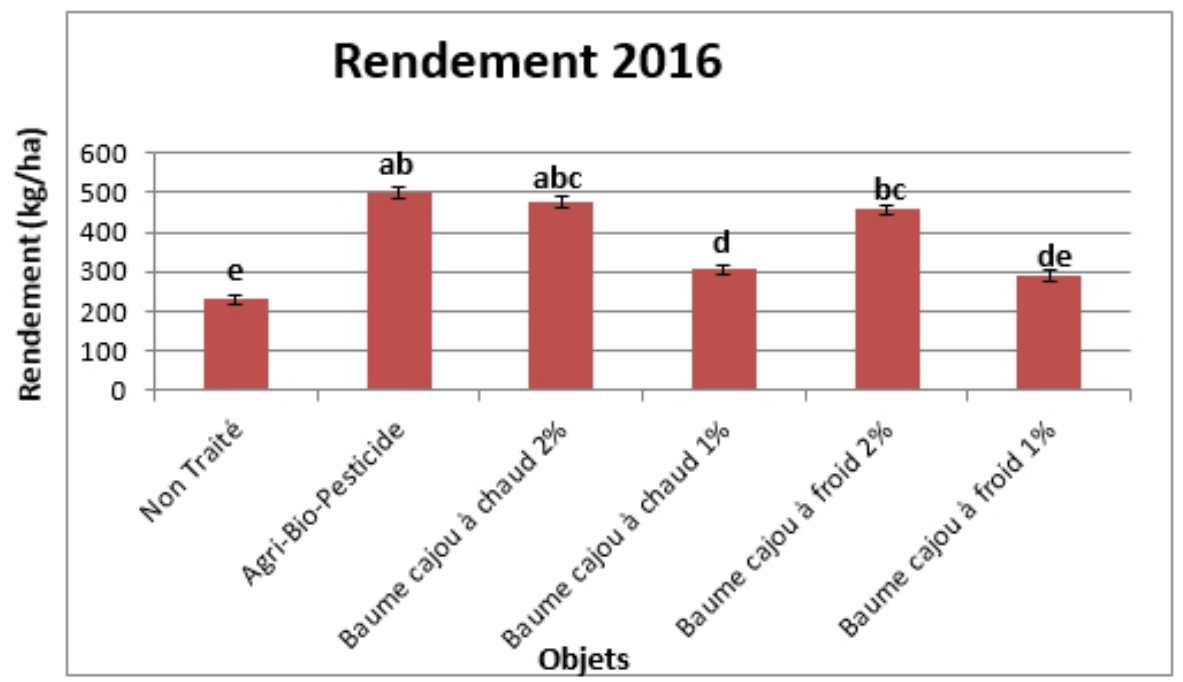

Figure 11 : Rendement en coton graine

\section{Discussion}

Le recours aux composés naturels issus des produits végétaux, et résultant du métabolisme secondaire des plantes, constitue une source potentielle de pesticides botaniques. Ces derniers sont facilement biodégradables, moins toxiques et ont moins d'impact possible sur l'environnement et la santé humaine (Regnault-Roger et al., 2008). Les résultats de l'évaluation de l'effet du Baume de cajou sur la densité des chenilles carpophages du cotonnier montrent qu'il y a une différence significative par rapport aux observations faites sur ces chenilles dans les parcelles non traitées qui ont hébergé plus de ravageurs que les parcelles traitées. Des résultats similaires ont été obtenus avec les extraits aqueux de substances épicées (Sinapis nigra L. [Brassicaceae], X. aethiopica, $N$. tabacum) sur les insectes ravageurs de Phaseolus vulgaris L. (Fabaceae) (Kambou et Guissou, 2011). En effet, ces auteurs ont montré qu'avec 400 1/ha d'extrait, ces substances épicées peuvent réduire de 61 à $78 \%$ la population des aleurodes, des thrips, des coléoptères, en comparaison aux parcelles témoins non traitées. De même, en traitant les parcelles de cultures (chou et gombo) avec l'extrait de graines de Azadirachta indica, Gnago et al. (2010) ont remarqué que les populations de chenilles et de pucerons étaient moins importantes sur les parcelles traitées que sur celles non traitées.

Le nombre de chenilles de $H$. armigera a été plus élevé en 2015 qu'en 2016. Le Baume de cajou extrait à froid comme à chaud dosé à $2 \%$ a eu la même performance sur les chenilles de $H$. armigera que le témoin de référence Agri-bio-pesticide. La dose de $1 \%$ du Baume de cajou extrait à chaud comme à froid serait donc insuffisant sur les chenilles de $H$. armigera en cas de forte infestation. Des résultats similaires ont été obtenus par Mochiah et al. (2011) 
qui ont constaté une baisse considérable des ravageurs du gombo et de l'aubergine (A. gossypii, Podagrica spp., B. tabaci, Earias spp., Dysdercus superstitiosus H. Schaffer, Z. variegatus L., Urentius hysterricellus Richter et Leucinodes orbonalis Guénée) après traitement avec des extraits d'Allium sp. (Liliaceae) (30 g.1-1) et de Carica papaya (92 g.1-1). De même, Solsoloy et al. (2000) ont conduit une étude pareille sur les ravageurs du cotonnier où les doses de 800 et $1250 \mathrm{ml} /$ ha d'huile de Jatropha curcas ont été comparées au profenofos à $400 \mathrm{~g} / \mathrm{ha}$ et à la deltamétrine à $12,5 \mathrm{~g} / \mathrm{ha}$. Ils ont retenu que les insecticides de synthèse ont été plus efficaces que l'huile de $J$. curcas sur $H$. armigera. Ils ont conclu que l'huile de Jatropha curcas a eu un effet plus lent que les produits de synthèse. De plus cette huile a agi sur la croissance des insectes.

Concernant le dénombrement des chenilles de Diparopsis watersi et de Earias spp., les deux concentrations de baume de cajou extrait à chaud comme à froid ont eu une efficacité similaire au témoin de référence Agri-BioPesticide durant les deux années d'expérimentation. L'effet insecticide des graines de neem et du piment contenus dans l'Agri-Bio-Pesticide a été montré par plusieurs auteurs. En effet, selon Deravel et al. (2013), l'huile extraite des graines de neem contient plusieurs molécules biologiquement actives dont l'azadirachtine, la nimbidine, la nimbidinine, la solanine, le déacétylazadirchtinol et le méliantriol. De plus, l'activité biocide des alcaloïdes, des saponines et des flavonoïdes extraits des fruits de Capsicum frutescens L. a été confirmée par Bouchelta et al. (2005). Yarou et al. (2017) ont révélé que Azadirachta indica est l'espèce sans doute la plus utilisée comme plante pesticide en Afrique de l'ouest. Siriwattanarungsee et al., 2008 ont constaté que l'Azadirachtine provoquait une perturbation de croissance chez les larves et une inhibition de la fécondité des mouches adultes. Des résultats similaires de forte mortalité adulticide de l'action des huiles de Azadirachta indica contre Anopheles gambiae, vecteur de paludisme ont été obtenus par Agbizounon (2010). Selon Chougourou et al. (2012), les traitements larvaires de mouche domestique, ont prouvé que les huiles de Azadirachta indica ont présenté une propriété larvicide très forte contre $M u s c a$ domestica au stade 3. Mais, plusieurs études ont montré que les composés de neem en général et des graines de cette espèce végétale en particulier, contenaient une quantité élevée d'azadirachtine, composé régulateur de la dynamique des insectes ravageurs des cultures et des stocks, mais aussi des insectes vecteurs (Liang et al., 2003 ; Aggarwal et Brar, 2006 ; Siddiqui et al., 2009; Degri et al. 2013; Shannag et al., 2014). Agboyi (2009) a révélé l'efficacité d'extrait de graines de neem sur Plutella xylostella et une espèce de puceron dans les cultures de chou au Togo. Les composés d'extraits de neem ont des effets régulateurs (antibioses et /ou anticénoses) sur les populations d'insectes ravageurs (Charleston et al., 2005 ; Mondédji et al., 
2014). Amtul (2014) a rapporté que Azadirachta indica renferme des composés agissant comme des inhibiteurs de l'enzyme digestive alphaamylase chez l'insecte ravageur Tribolium castaneum (Coleoptera : Tenebrionidae).

La performance de l'Agri-bio-pesticide résulte également du piment Capsicum frutescens L. qu'il contient dont les fruits contiennent des alcaloïdes, des saponines et des flavonoïdes. En effet, Bouchelta et al. (2005) ont montré que ces trois composés du piment, affectent significativement la survie des stades de développement de Bemisia tabaci infestant les plants de tomates selon la concentration et la durée d'exposition. D'un point de vue chimique, en plus des composés primaires, le genre Capsicum contient divers métabolites secondaires tels que des alcaloïdes sous formes de capsaicidine (Saber, 1976), de capsaicine (Cordell et Araujo, 1993) et de solanine (Newall et al., 1996), des saponines (De Lucca et al., 2002) et des flavonoïdes avec d'autres composés phénoliques (Materska et al., 2003 ; Zang et Hamauru, 2003). En protection des plantes, la poudre et les extraits de Capsicum frutescens ont montré un pouvoir répulsif contre Callosobruchus maculatus (F.) (Zibokere, 1994 ; Onu et Aliyu, 1995 ; Ofuya, 1986), Rhyzopertha dominica (L.) (El-Lakwah et al., 1997), Sitophilus zeamaïs Motsch et Tribolium castaneum (Herbst) (Morallo-Rejesus, 1987 ; Trematerre et Sciarretta, 2002). La toxicité des extraits des fruits du piment fort a aussi été notée chez Rhyzopertha dominica, S. oryzae (L.) et T. confusum J. du Val (Williams et Mansingh, 1993 ; Gakuru et Foua, 1996). Dans les conditions d'étude, les alcaloïdes, les saponines et les flavonoïdes, extraits des fruits de Capsicum frutescens, affectent la viabilité des œufs et la survie des adultes de Bemisia tabaci (Bouchelta et al., 2005). Comme cela a été observé chez Trialeurodes vaporariorum (Choi et al., 2003), les alcaloïdes se sont avérés plus efficaces que les saponines et les flavonoïdes. Les alcaloïdes peuvent avoir des effets toxiques par contact chez les embryons, par contact et/ou par ingestion chez les adultes de Bemisia tabaci. Les extraits aqueux de Capsicum frutescens L. contiennent des substances allélochimiques agissant par des effets répulsifs, antiappétant ou toxiques contre les phytophages généralistes (Ehrlich et Raven, 1964). La poudre et les extraits de C. frutescens ont un effet répulsif sur Sitophilus zeamaïs Motsch et Tribolium castaneum (Herbst) (Trematerra et Sciarretta, 2002). Al-Moajel (2004) a aussi montré que les extraits de Capsicum frutescens ont un effet toxique sur les larves de Trogoderma granarium.

Par ailleurs, le Baume de cajou extrait à froid comme à chaud dosé à $2 \%$ a eu la même performance sur les chenilles carpophages que le témoin de référence Agri-bio-pesticide. Plusieurs travaux ont confirmé l'activité biocide du baume de cajou. En effet, Selon Araùjo et Xavier (2009) tous les deux types de baume (extrait à froid ou à chaud) comportent principalement de l'acide 
anacardique, du cardanol, du cardol, du 2-méthylcardol à des proportions différentes. Kpoviessi et al., (2017) ont montré que le baume de cajou peut réduire significativement les populations des nuisibles (pucerons, thrips et Maruca) du niébé. Compte tenu des métabolites secondaires des plantes, de nombreux travaux ont mis en évidence les effets dépressifs des extraits de plantes sur des ravageurs phytophages; ces composés (ou substances allélochimiques) sont importants dans les interactions de médiation entre les plantes et leur environnement biotique (Kessler et Baldwin, 2002). Nos résultats indiquent que le mode d'extraction n'a pas influencé la performance du baume sur les chenilles carpophages du cotonnier. Mais ces résultats sont contraires à ceux obtenus par Akpo (2017) qui a déduit de ses études que le baume de cajou est plus efficace quand on l'extrait à froid qu'à chaud sur les larves de moustique qui y sont exposées.

Les résultats de dénombrement des capsules (vertes ou mûres) a révélé au niveau du pourcentage de capsules saines et trouées que les différentes concentrations de Baume de cajou ont eu la même performance que le témoin de référence, Agri-bio-pesticide durant les deux années d'expérimentation. Le mode d'extraction n'a pas influencé la performance du Baume de cajou tel que constaté au niveau des chenilles. Ces résultats sont similaires à ceux obtenus par Asare-Bediako et al. (2014) qui ont montré la capacité des extraits de Azadirachta indica, de Carica papaya, d'Allium sp., de Capsicum sp. (Solanaceae), de Anacardium sp. (Anacardiaceae) à minimiser la sévérité de la virose due aux aleurodes du gombo.

Les résultats de rendement de coton graine ont révélé que l'Agri-biopesticide, le Baume de cajou extrait à chaud dosé à $2 \%$ et le Baume de cajou extrait à froid dosé à $2 \%$ ont été les meilleurs biopesticides. La concentration de $1 \%$ de Baume de cajou extrait à chaud comme à froid a permis d'obtenir des rendements inférieurs au témoin de référence Agri-bio-pesticide. La tendance selon laquelle la concentration de $1 \%$ de Baume de cajou extrait à chaud comme à froid serait insuffisante en cas de forte infestation a été confirmé au niveau du rendement. On constate aussi que les rendements moyens des parcelles traitées sont significativement supérieurs à ceux des parcelles non traitées. Ces rendements sont en concordance avec les situations d'infestations et des taux de dégâts observés. Ces résultats sont similaires à ceux obtenus par Kpoviessi et al., (2017) qui ont montré que le baume de cajou a favorisé une augmentation significative du rendement du niébé. Des résultats similaires ont été obtenus par Mondedji et al. (2014) qui a constaté que les parcelles traitées avec les extraits de feuilles de Azadirachta indica ont donné les meilleurs rendements en chou commercialisable. De même, Kambou et Guissou (2011) ont montré que l'utilisation de 400 1/ha d'extrait aqueux de substances épicées (Sinapis nigra L. [Brassicaceae], X. aethiopica, $N$. tabacum) permet d'assurer un rendement de haricot vert équivalent à celui 
obtenu sur les parcelles traitées avec un insecticide de synthèse comme la deltaméthrine. Asare-Bediako et al. (2014) ont montré la capacité des extraits de Azadirachta indica, de Carica papaya, de Allium sp., de Capsicum sp. (Solanaceae), de Anacardium sp. (Anacardiaceae) à augmenter le rendement de gombo des parcelles traitées.

\section{Conclusion}

La présente étude a permis de disposer de connaissances scientifiques sur l'efficacité du Baume de cajou sur les ravageurs carpophages en culture cotonnière biologique au Bénin. Cette étude a montré que le Baume de cajou a un effet régulateur sur les populations de chenilles carpophages du cotonnier. Le mode d'extraction n'influence pas l'efficacité du Baume de cajou sur les ravageurs carpophages du cotonnier. La concentration de $1 \%$ du Baume de cajou a été insuffisante pour contrôler les ravageurs carpophages en cas de forte infestation. Mais celle de $2 \%$ de baume de cajou a été autant efficace que le témoin de référence Agri-bio-pesticide utilisé par la grande partie des producteurs de coton biologique au Bénin. Cette capacité du Baume de cajou à réduire le nombre de ravageurs carpophages et leurs dégâts, a permis l'obtention des rendements équivalents au témoin de référence. Ces résultats ont montré que le Baume de cajou possède des propriétés insecticides. Il se positionne comme une alternative aux biopesticides utilisés en culture cotonnière biologique. Il serait un produit phytosanitaire sans impact négatif sur la santé humaine et sur les animaux et peut contribuer à la sauvegarde et à la protection de l'environnement. En perspective, il serait important de quantifier la teneur des molécules insecticides contenues dans le Baume de cajou puis de formuler un biopesticide à l'usage des producteurs.

\section{References:}

1. Agbizounon A., 2010. Tests d'efficacité d'extraits d'huiles végétales sur Anopheles gambiae Giles et Culex quinquefasciatus Say résistants aux pyréthrinoïdes. Mémoire Ingénieur de conception, EPAC Abomey-Calavi, Bénin. 51p.

2. Agboyi L. K. B. A., 2009. Vulnérabilité des agroécosystèmes maraîchers du Togo et essai de biocontrôle de deux ravageurs Brevicoryne brassicae et Plutella xylostella sur le chou à l'aide de Beauveria bassiana 5653 et de l'extrait aqueux d'amandes de graines de neem (Azadirachta indica A. Juss). Mémoire de D.E.A., Université de Lomé, 61p.

3. Aggarwal N., Brar D. S., 2006. Effects of different neem preparations in comparison to synthetic insecticides on the whitefly parasitoid Encarsia Sophia (Hymenoptera : Aphelinidae) and the predator 
Chrysoperla carnea (Neuroptera: Chrysopidae) on cotton under laboratory conditions. Journal of Pest Science, 79(4): 201-207.

4. Akpo A. A., 2017. Evaluation de l'efficacité des extraits des Plantes locales pour le contrôle des vecteurs du paludisme résistants aux pyréthrinoïdes au Bénin (Afrique de l'Ouest). Thèse de Doctorat de l'Université d'Abomey-Calavi. 253p.

5. Al-Moajel N. H., 2004. Testing some various powders for protection of wheat grain against Tropodermagranarium Everts. Journal of Biological Sciences 4 : 592-7.

6. Amtul J. S., 2014. Azadirachta indica derived compounds as inhibitors of digestive alpha-amylase in insect pests : Potential bio-pesticides in insect pest management. Europ. J. Exp. Biol., 4(1): 259-264.

7. Araújo da Silva F. J., Xavier de Matos J. E., 2009. A note on the potential of CNSL in fuel blends for engines in Brazil. Rev. Tecnol., Fortaleza 30, 89-96.

8. Asare-Bediako E., Addo-Quaye A., Bi-Kusi A., 2014. Comparative efficacy of plant extracts in managing whitefly (Bemisia tabaci Gen.) and leaf curl disease in okra (Abelmoschus esculentus L.). Am. J. Agric. Sci. Technol., 2(1), 31-41.

9. Baffes J., 2007. The "cotton problem" in west and central Africa: The case for domestic reforms. CATO Institue, Econ. Dev. Bull. $\mathrm{N}^{\circ} 11$.

10. Bolker B., Skaug H., Magnusson A., Nielsen, A., 2012. Getting started with the glmmADMB package. Retrieved from http://glmmadmb.rforge.r-project.org/glmmADMB.html

11. Bouchelta A., Boughdad A., Blenzar A., 2005. Effets biocides des alcaloïdes, des saponines et des flavonoïdes extraits de Capsicum frutescens L. (Solanaceae) sur Bemisia tabaci (Gennadius) (Hom ; Aleyrodidae). Biotechnology, Agronomy, Society and Environment 9 :259-269.

12. Burnham K. P., Anderson D. R., 2002. Model selection and multimodel inference: a practical information-theoretic approach, 2nd edn. Springer, New York.

13. Cavalcante A. A., Rübensam G., Picada J., Silva E., Moreira F., Henriques J., 2003. Mutagenic evaluation, antioxidant potential and antimutagenic activity against hydrogen peroxide of cashew (Anacardium occidentale L.) apple juice and cajuina. Environ. Mol. Mut 41: 360-369.

14. Chabi S. K., Adoukonou-Sagbadja H., Ahoton L. E., Adebo I., Adigoun F.A., Saidou A., Kotchoni S. O., Ahanchede A., BabaMoussa L., 2013. Indigenous knowledge and traditional management of cashew (Anacardium occidentale L.) genetic resources in Benin. 
Journal of Experimental Biology and Agricultural Sciences 1: 375382.

15. Chabi, S. K., Adoukonou-Sagbadja H., Ahoton L. E., Roko G. O., Saidou A., Adeoti K., Ahanchede A., Baba-Moussa L., 2014. Antimicrobial activity of Anacardium occidentale L. leaves and barks on pathogenic bacteria. African Journal Microbiology research 8: 2458-2467.

16. Charleston D. S., Kfir R., Lem V., Dicke M., 2005. Behavioural responses of diamondback moth Plutella xylostella (Lepidoptera: Plutellidae) to extracts derived from Melia azedarach and Azadirachta indica. Bulletin of Entomological Research, 95: 457-465.

17. Choi W. I., Lee E. H., Choi B. R., Park H. M., Ahn Y. J., 2003. Toxicity of plant essential oils to Trialeurodes vaporariorum (Homoptera: Aleyrodidae). J. Econ. Entomol. 96 (5), p. 1479-1484.

18. Chougourou C. D. ; Dellouh P. L., Agbaka A., N'Guessan K. R., Gbenou J. D., 2012. Toxicité et effets répulsifs de certaines huiles extraites des plantes locales Béninoises sur la mouche domestique Musca domestica L. (Diptera Muscidae). Journal of Applied Biosciences 55: 3953 - 3961.

19. Cordell G. A., Araujo O. E., 1993. Capsaicin: identification, nomenclature and pharmacotherapy. Ann. Pharmacother. 27, p. 383395.

20. Degri M. M., Mailafiya D. M., Wabekwa J. W., 2013. Efficacy of aqueous leaf extracts and synthetic insecticide on pod-sucking bugs infestation of cowpea (Vigna unguiculata (L.) Walp) in the Guinea Savanna Region of Nigeria. Advances in Entomology, 1(2): 10-14.

21. De Lucca A. J., Bland J. M., Vigo C. B., Cushion M., Selitrennikoff C. P., Peter J., Walsh T. J., 2002. CAY -I. a fungicidal saponin from Capsicum sp. fruit. Med. Mycol. 40 (2), p. 131-137.

22. Deravel J., Krier F., Jacques P., 2013. Les biopesticides, compléments et alternatives aux produits phytosanitaires chimiques (synthèse bibliographique). Biotechnol. Agron. Soc. Environ. 18(2), 220-232.

23. Diakite L., Djouara H., 2003. Etude socio-économique de la production du coton biologique 2002 - 2003 au Mali. Ministère de l'Agriculture de l'Elevage et de la Pêche, Institut d'Economie Rurale, Programme Economie des Filières. 48 p.

24. Ehrlich P. R., Raven P. H., 1964. Butterflies and plant: a study in coevolution. Evolution 18 : 586-608.

25. EL-Lakwah F., Khaled O. M., Kattab M. M., Abdel- Rahman T. A., 1997. Effectiveness of some plant extracts and powders against the lesser grain borer Ryzopertha dominica (F.). Ann. Agric. Sci. 35 (1), p. 567-578. 
26. FAO, 2014. Gestion intégrée de la production et des déprédateurs du coton. Guide du facilitateur pour les champs écoles des producteurs. 89p.

27. Ferrigno S., Lizarraga A., 2009. Les composants d'un système de production durable du coton : Perspectives suivant l'expérience du coton organique. ICAC RECORDER. 14-24.

28. Ferron P, Deguine J. P., Mouté J. E. M., 2006. Évolution de la protection phytosanitaire du cotonnier: un cas d'école. Cah. Agric., 15(1): 128-134.

29. Fox J., Weisberg S., 2011. An $\{\mathrm{R}\}$ Companion to Applied Regression, Second Edition. Thousand Oaks CA: Sage. URL: http://socserv.socsci.mcmaster.ca/jfox/Books/Companion

30. Gakuru S., Foua B. K., 1996. Effects of plant extracts on the cowpea weevil (Callosobruchus maculatus Fab.) and the rice weevil (Sitophilus oryzae L.). Cah. Agric. 5 (1), p. 39-42.

31. Gnago A. J., Danho M., Atcham Agneroh T., Fofana K. I., Kohou G. A., 2010. Efficacité des extraits de neem (Azadirachta indica) et de papayer (Carica papaya) dans la lutte contre les insectes ravageurs du gombo (Abelmoschus esculentus) et du chou (Brassica oleracea) en Côte d'Ivoire. Int. J. Biol. Chem. Sci. 4(4): 953-966.

32. Hougni A., Kpadé P. C., Djihinto A. C., 2012. Culture biologique du cotonnier et commerce équitable du coton : Deux approches de niche. Bulletin de la Recherche Agronomique du Bénin (BRAB). Numéro spécial Coton. 60-74

33. Kambou G., Guissou I. P., 2011. Phytochemical composition and insecticidal effects of aqueous spice extracts on insect pests found on green beans (Phaseolus vulgaris) in Burkina Faso. Tropicultura, 29(4), 212- 217.

34. Kessler A, Baldwin I. T., 2002. Plant responses to insect herbivory : The emerging molecular analysis. Annual Review of Plant Biology 53: 299-328.

35. Kpoviessi A. D., Dossou J., Chougourou C. D., Bokonon-Ganta H. A., Francisco A. R., Fassinou-Hotegni V. N., 2017. Evaluation de 1'effet insecticide et insectifuge du Baume de Cajou sur les insectes nuisibles du niébé Vigna unguiculata (L.) Walp. au Champ. European Journal of Scientific Research 146 (4), 417 - 432.

36. Kranthi K. R., Jadhav D., Wanjari R., Kranrhi S., Russel D., 2001. Pyrethroid resistance and mechanisms of resistance in field strains of Helicoverpa armigera (Lepidoptera: Noctuidae). Journal Econ. Entomol., 94(1): 253-263. 
37. Liang G.-M., Chen W., Liu T. X., 2003. Effects of three neem-based insecticides on diamondback moth (Lepidoptera: Plutellidae). Crop Protection., 22: 333-340.

38. Materska M., Piacenteb S., Stochmalc A., Pizzab C., Oleszekc W., Peruckaa I., 2003. Isolation and structure elucidation of flavonoid and phenolic acid glycosides from pericarp of hot pepper fruit Capsicum annuum L. Phytochemistry 63 (8), p. 893-898.

39. Matthews G. A, 1989. Cotton insect pests and their management. Longnian Scientific and Technical, New York (USA), 199 p.

40. Mochiah M., Banful B., Fening K., 2011. Botanicals for the management of insect pests in organic vegetable production. $J$. Entomol. Nematol., 3, 85-97.

41. Mondedji A. D., Ketoh G. K., Amévoin K., Améline A., Giordanengo P., Glitho I. A., 2014. Evaluation of neem leaves-based preparations as insecticidal agents against the green peach aphid, Myzus persicae (Sternorrhyncha : Aphididae). African Journal of Agricultural Research, 9(17): 1344-1352.

42. Morallo-Rejesus B., 1987. Botanical pest control research in the Philippines. Philipp. Entomol. 7, p. 1-30.

43. Moseley W., Gray L. C., 2008. Hanging by a thread: cotton, globalization and poverty in Africa. Othio University Press, Athens, $\mathrm{OH}$.

44. Newall C. A., Anderson L. A., Philipson J. D., 1996. Herbal Medicines: a Guide for heath care professionals. London, England: The pharmaceutical Press. p. 44.

45. OBEPAB, 2002. Le Coton au Bénin: rapport de consultation sur le coton conventionnel et le coton biologique au Bénin, 36p.

46. Ofuya T. L., 1986. Use of word ash, dry chilli pepper fruits and onion scale leaves for reducing Callosobruchus maculatus (Fabricius) damage in cowpea seeds during storage. J. Agr. Sci. 107 (2), p. $467-$ 468.

47. Onu I., Aliyu M., 1995. Evaluation of powdered fruits of four peppers (Capsicum spp.) for the control of Callosobruchus maculatus (F.) on stored cowpea seed. Int. J. Pest Manag. 41 (3), p. 143-145.

48. Pinheiro J., Bates D., DebRoy S., Sarkar D., R Core Team., 2017. _nlme: Linear and Nonlinear Mixed Effects Models_. R package version 3.1-131

49. R Development Core Team., 2017. R: a language and environment for statistical computing. R Foundation for Statistical Computing, Vienna, Austria. www.r-project.org 
50. Regnault-Roger C., Philogène B. J. R., Vincent C., eds, 2008. Biopesticides d'origine végétale. 2e édition, Paris : Lavoisier Tech \& Doc.

51. Russell V. L., 2016. Least-Squares Means: The R Package lsmeans. Journal of Statistical Software, 69(1), 1-33. doi:10.18637/jss.v069.i01

52. Saber M. S., 1976. Antimicrobial substance in certain members of Solanaceae. IV. Detection of active principle in pepper. Zentralbl. Bakteriol. Parasitenkd Infektionkr. Hyg. 131 (2), p. 110-112.

53. Shannag H. S., Capinera J. L., Freihat N. M., 2014. Efficacy of different neem-based biopesticides against green peach aphid, Myzus persicae (Hemiptera: Aphididae). International Journal of Agricultural Policy and Research, 2(2): 061-068.

54. Siddiqui B. S., Ali S. K., Ali S. T., Naqvi S. N. U., Tariq R. M., 2009. Variation of major limonoids in Azadirachta indica fruits at different ripening stages and toxicity against Aedes aegypti. Nat. Prod. Commun., 4: 473-476.

55. Siriwattanarungsee S., Kabkaew L., Olson K., Chailapakul O., Sukontason K., 2008. Efficacy of neem extract against the blowfly and housefly. Parasitol. Res. 103: 535-544.

56. Solsoloy A. D., Domingo E. O., Cacayorin M. D., Damo M. C., 2000. Chemical insecticides for cotton pest control. In: Proceedings of Regional Research and Development Symposia, Jul.-Sep. 1999. Los Banos, Laguna, Philippines: Philippine Council for Agriculture, Forestry and Natural Resources Research and Development.

57. Tovignan S., 2012. Production de fibre de coton biologique et bioéquitable : Rapport pour l'Afrique 2010-2011. Textile Exchange. 7 p.

58. Trematerra P., Sciarretta A., 2002. Activity of chilli, Capsicum annuum L. var. acuminatum, on stored product insects Oryzaephilus surinamensis (L.), Sitophilus oryzae (L.) and Tribolium castaneum (Herbst). IOBC/wprs Bull. 25 (3), p. 177-182.

59. Venables W. N., Ripley B. D., 2002. Modern Applied Statistics with S. Fourth Edition. Springer, New York. ISBN 0-387-95457-0

60. Williams L. A. D., Mansingh A., 1993. Pesticidal potential of tropical plants - I. Insecticidal activity of leaf extracts of sixty plants. Insect Sci. Applic. 14 (5), p. 697-700.

61. Yarou B. B., Silvie P., Assogba Komlan F., Mensah A., Alabi T., Verheggen F., Francis F., 2017. Plantes pesticides et protection des cultures maraichères en Afrique de l'Ouest (synthèse bibliographique). Biotechnol. Agron. Soc. Environ. 21(4), 288-304.

62. Zang D., Hamauru Y., 2003. Phenolic compounds, ascorbic acid, carotenoids and antioxidant properties of grebe, red and yellow bell peppers. Food, Agric., Environ. 1 (2), p. 22-27. 
63. Zibokere D. S., 1994. Insecticidal potency of red pepper (Capsicum annum) on pulse beetle (Callosobruchus maculatus) infesting cowpea (Vigna unguiculata) seeds during storage. Indian J. Agr. Sci. 64 (10), p. 727-728. 